\title{
Prepare for a pandemic
}

\author{
Richard Hurley features and debates editor
}

The BMJ

A covid-19 pandemic is highly likely, warns our editorialist, the epidemiologist John Watkins (doi:10.1136/bmj.m810), and we need to plan now how to reconfigure care services to cope with the looming escalation in demand. "We should plan on the assumption that most of the population may contract the virus with few or no long term effects," he writes, "while harnessing vital secondary healthcare resources to treat the small percentage of people who become seriously ill."

As at Tuesday 3 March the UK had confirmed 51 cases of infection (doi:10.1136/bmj.m854). England's chief medical officer, Chris Witty, said at the launch of the government's plan that at worst $80 \%$ of people could be infected, most with mild symptoms. He suggested a $1 \%$ fatality rate, but with many more deaths among older people and patients with pre-existing conditions.

The government's response remains focused on containment to prevent transmission. Watkins cautions that attempts in 2009 to contain the global spread of H1N1 influenza failed, however. Although containment "can buy us some time," he acknowledges, "containment in the face of a pandemic will go only so far, and we must use this time wisely."

Stretched NHS and social care services, he says, "will be severely challenged even by a modest increase in cases."

The government's plan, scant in detail and lacking a timescale, mentions options to delay non-urgent care and to vary staff rostering, including recalling retired doctors and those on leave.
It also mentions stockpiling drugs and equipment and recognises that staff welfare, given personal illness and caring responsibilities, will be "critical to supporting an extended response."

Worldwide, some 90000 people have been infected in more than 60 countries, with about 80000 in China. More than 3000 have died. Italy is Europe's worst affected country, with at least 1835 people having been infected and 52 deaths, and Fabrizio Carinci's editorial unpicks the country's experience (doi:10. 1136/bmj.m799). For all The BMJ's covid-19 coverage, free to access, see bmj.com/coronavirus.

Elsewhere on bmj.com this week, a meta-analysis concludes that moderate egg consumption is not associated with overall cardiovascular risk (doi:10.1136/bmj.m513). "We should not put all our eggs in this observational basket" for formal dietary guidance, says Andrew Odegaard in his linked editorial, however (doi:10.1136/bmj.m744). "The best evidence for cardioprotection supports shifting one's overall dietary pattern," to a Mediterranean diet, for example.

And our latest Head to Head debate asks whether big pharma should be state run (doi:10.1136/bmj.m769). Mariana Mazzucato and Henry Lishi Li point to the industry's "short termism and misalignment with public interest." Ara Darzi acknowledges the problems but argues they can be managed so that drug firms can continue to make "transformative improvements in global health." 